\title{
NOTE
}

\section{Alpha Linolenic Acid-enriched Diacylglycerol Consumption Enhances Dietary Fat Oxidation in Healthy Subjects: A Randomized Double-blind Controlled Trial}

\author{
Yasutoshi Ando', Shinichiro Saito ${ }^{1 *}$, Nami Yamanaka1, Chizuka Suzuki ${ }^{1}$, Takahiro Ono², \\ Noriko Osaki ${ }^{1}$ and Yoshihisa Katsuragi ${ }^{1}$ \\ ${ }^{1}$ Healthcare Food Research Laboratories, Kao Corporation 2-1-3 Bunka Sumida-ku Tokyo 131-8501, JAPAN \\ ${ }^{2}$ Medical Corporation Wakei-kai Medics Hongo Clinic, Bunkyo-ku, Tokyo 113-0023, JAPAN
}

\begin{abstract}
Consumption of alpha linolenic acid-enriched diacylglycerol (ALA-DAG) reduces visceral fat area. In this study, we performed a randomized, placebo-controlled, double-blind, crossover intervention trial to investigate the effect of ALA-DAG on dietary fat oxidation in comparison with control triacylglycerol (TAG). Each subject $(n=16)$ consumed either $2.5 \mathrm{~g} / \mathrm{d}$ of ALA-DAG or TAG for 14-d, separated by a 21-d washout period. At the end of each consumption period, we assessed dietary fat oxidation. ALA-DAG consumption significantly enhanced dietary fat utilization as energy compared to TAG consumption.
\end{abstract}

Key words: ALA-DAG, alpha-linolenic acid, diacylglycerol, dietary fat oxidation, human

\section{INTRODUCTION}

Visceral obesity is strongly associated with metabolic risk factors, such as hyperglycemia, hypertension, and hyperlipidemia $^{1-3)}$. The development of obesity is related to genetic background, physical activity, and diet composition. In particular, a chronic imbalance between fat intake and fat expenditure is an important regulator of body fat ${ }^{4)}$. Moreover, dietary fat ingestion may be related to fat oxida$\operatorname{tion}^{5-7)}$

Alpha linolenic acid-enriched diacylglycerol(ALA-DAG) is a minor natural component of many edible oils and has long been consumed by humans. ALA-DAG mainly occurs with the chemical structure 1,3-diacyl-sn-glycerol (1,3$\mathrm{DAG}$ ) and alpha-linolenic acid as the fatty acid. Previous human studies demonstrated that the long-term consumption of ALA-DAG significantly decreases body weight and visceral fat area compared to consuming the control triacylglycerol $(\mathrm{TAG})^{8-10)}$. ALA-DAG consumption enhances both fat oxidation and energy expenditure in healthy humans ${ }^{11)}$. Thus, ALA-DAG consumption could be useful for controlling body weight by maintaining or improving fat and energy metabolism. This underlying mechanism is supported by reports showing enhanced expression of $\beta$-oxidation related enzymes and genes in the small intestine ${ }^{12)}$ and in the liver ${ }^{13)}$ in rodents. Based on these reports, ALA-DAG is expected to enhance oxidation of diet-derived fat as well as body fat. Indeed, in rodents, dietary fat oxidation is enhanced after repeated ingestion of ALA-DAG compared to $\mathrm{TAG}^{14)}$. Its effect in humans, however, remains unclear. Therefore, this study evaluated whether repeated ALA-DAG consumption affects oxidation of diet-derived fat in healthy human subjects.

\section{EXPERIMENTAL}

\subsection{Ethics and registration}

This study was performed in accordance with the principles of the Declaration of Helsinki and was approved by the clinical ethics committee of Oriental Ueno Kenshin Center (Tokyo, Japan). The study protocol was registered in advance with the University hospital Medical Information

\footnotetext{
*Correspondence to: Shinichiro Saito, Healthcare Food Research Laboratories, Kao Corporation, 2-1-3 Bunka Sumida-ku Tokyo 131-8501, JAPAN

E-mail: saito.shinichiro@ kao.co.jp

Accepted September 25, 2016 (received for review September 15, 2016)
}

Journal of Oleo Science ISSN 1345-8957 print / ISSN 1347-3352 online

http://www.jstage.jst.go.jp/browse/jos/ http://mc.manusriptcentral.com/jjocs 


\section{Y. Ando, S. Saito and N. Yamanaka et al.}

Network Clinical Trials Registry(registration no. UMIN000021181), and the execution of this study was outsourced to TES Holdings Co., Ltd (Tokyo, Japan). After receiving an explanation of the study, all subjects provided written informed consent to participate in the study.

\subsection{Subjects}

Twenty subjects were enrolled in the study according to the inclusion and exclusion criteria. Inclusion criteria were 1) age 35 to 64 years and 2) body mass index 23.0 to 29.9 $\mathrm{kg} / \mathrm{m}^{2}$. Exclusion criteria included the presence of severe disease, surgery within 2 months, taking medication, taking supplements or foods with health claims, premenopausal woman, and food allergy. The sample size was estimated based on our previous study showing that ALA-DAG significantly enhanced postprandial total fat oxidation in 19 subjects $^{11)}$. Because the dose and the consumption duration of ALA-DAG in this present study were the same as the previous study, we estimated that a similar sample size was appropriate.

\subsection{Design and protocol}

The study had a randomized, double-blind, placebo-controlled crossover design with two 14-d consumption periods of ALA-DAG or control TAG, separated by a 21-d washout period. The subjects were assigned to each order of the consumption period by stratified block randomization using computer-generated random numbers under blinded condition. During each consumption period, the subjects consumed shortbreads containing $2.5 \mathrm{~g} / \mathrm{d}$ of ALA-DAG or TAG each day. During the consumption period, the subjects were instructed to maintain their habitual lifestyle, including their usual physical activity and dietary intake. The subjects recorded their meals for 3-d before the measurements, and nationally registered dietitians analyzed the dietary records. Alcohol intake and strenuous exercise were not allowed for the 3-d before each measurement. One day before the measurements, the subjects ingested specified meals for breakfast, lunch, and dinner (total calories: $2173 \mathrm{kcal} /$ day for men, $1818 \mathrm{kcal} /$ day for women). At the end of the consumption period, we measured the subjects' body composition and dietary fat oxidation, and collected serum samples after they had fasted for at least $12 \mathrm{~h}$.

\subsection{Test diet}

We used a previously reported method $^{15)}$ to prepare the ALA-DAG from flaxseed oil (Summit Oil Corporation, Chiba, Japan) and rapeseed oil (The Nisshin OilliO Group, Ltd, Tokyo, Japan) using equipment owned by Kao Corporation(Tokyo, Japan). Each $2.5 \mathrm{~g}$ of ALA-DAG contained $0.9 \mathrm{~g}$ DAG-bound ALA. Rapeseed oil was used as the source of the TAG (The Nisshin OilliO Group, Ltd, Tokyo, Japan). Table 1 shows the components of the TAG and
Table 1 Glycerides and fatty acids composition of TAG and ALA-DAG.

\begin{tabular}{lrc}
\hline & TAG & ALA-DAG \\
\hline Glyceride $(\mathrm{g} / 100 \mathrm{~g})$ & & \\
DAG & 1.5 & 80.2 \\
DAG-bound ALA & 0.1 & 35.3 \\
Monoacylglycerol & 0.0 & 0.5 \\
Free fatty acid & 0.0 & 0.1 \\
TAG and others & 98.5 & 19.4 \\
Fatty acid (wt, \%) & & \\
C16:0 & 4.1 & 2.6 \\
C18:0 & 1.9 & 1.5 \\
C18:1 & 61.0 & 26.9 \\
C18:2 & 20.4 & 16.9 \\
C18:3 & 9.3 & 50.7 \\
C20:0 & 0.6 & 0.1 \\
C20:1 & 1.1 & 0.4 \\
C22:0 & 0.4 & 0.3 \\
Others & 1.1 & 0.8 \\
\hline
\end{tabular}

ALA-DAG. We produced a cooking oil by mixing the ALA-DAG with rapeseed oil, anti-oxidants, and emulsifying agents. We made a test shortbread (hard flour, soft flour, superfine sugar, salt, egg, pullulan, water, and the prepared cooking oil) to ensure accurate ingestion of the ALA-DAG or TAG. Each shortbread (60 g) contained $291 \mathrm{kcal}$ (protein:fat:carbohydrate $=8: 39: 53)$ per $\operatorname{serving}(2.5 \mathrm{~g}$ ALA-DAG or TAG) and were individually packaged. The shortbreads containing TAG and ALA-DAG could not be distinguished from each other by appearance, taste, or odor.

\subsection{Dietary fat oxidation assessment}

We synthesized the ${ }^{13} \mathrm{C}$-labelled triolein probes from $\left[1-{ }^{13} \mathrm{C}\right]$ oleic acid (purity $>99 \%,{ }^{13} \mathrm{C}>99 \%$; Isotec, Miamisburg, OH, US) and free glycerol using an enzymatic method and purified the probes using silica gel liquid chromatography. The assessment of dietary fat oxidation was performed as reported previously ${ }^{16-18)}$. Briefly, before and after ingestion of the test meal $(555 \mathrm{kcal}$, protein: fat: carbohydrate $=$ 17:30:52 as energy value) containing ${ }^{13} \mathrm{C}$-labelled triolein (400 mg), we collected breath samples in aluminum bags (GL Science Inc., Tokyo, Japan) every hour for $6 \mathrm{~h}$. As the primary outcome, oxidation of dietary fat was assessed by measuring recovery rate of ingested ${ }^{13} \mathrm{C}$-labelled triolein to ${ }^{13} \mathrm{CO}_{2}$ in the breath. Recovery rate of ${ }^{13} \mathrm{C}$ was assessed by the combined use of an indirect calorimeter (Arco Systems Inc., Chiba, Japan) and an stable isotope ratio mass spectrometer (ANCA-GSL; Sercon, Crewe, UK). 


\subsection{Statistical analysis}

Data are expressed as mean \pm SD. Comparisons of the difference in two periods between the ALA-DAG treatment followed by the TAG treatment and the TAG treatment followed by the ALA-DAG treatment were performed using a two-sample t-test. A p-value of less than 0.05 was considered statistically significant.

\section{RESULTS}

\subsection{Subjects and characteristics}

Sixteen subjects ( 11 men and 5 women) completed the measurements and were included in the analyses, and 4 subjects did not complete the measurements. The baseline physical characteristics, including body composition, serum triglyceride, glucose, insulin, non-esterified fatty acid, total-, low-density-, and high-density-cholesterol did not significantly differ between the treatment orders. Table 2 shows the physical characteristics of the subjects after the treatments. Body fat ratio and fat mass were significantly lower after the ALA-DAG treatment compared with the TAG treatment. The serum parameters did not significantly differ after the treatments between ALA-DAG and TAG(data not shown).

\subsection{Outcomes}

The ${ }^{13} \mathrm{C}$ recovery at the $1 \mathrm{~h}$ time-point (Fig. 1A) and the cumulative ${ }^{13} \mathrm{C}$ recovery at $6 \mathrm{~h}$ (Fig. 1B) were significantly higher in the ALA-DAG treatment compared to the TAG treatment. The cumulative recovery of ${ }^{13} \mathrm{C}$ was $14.8 \pm 4.3 \%$ in the TAG treatment and $17.1 \pm 4.0 \%$ in the ALA-DAG treatment(Fig. 1B).

A

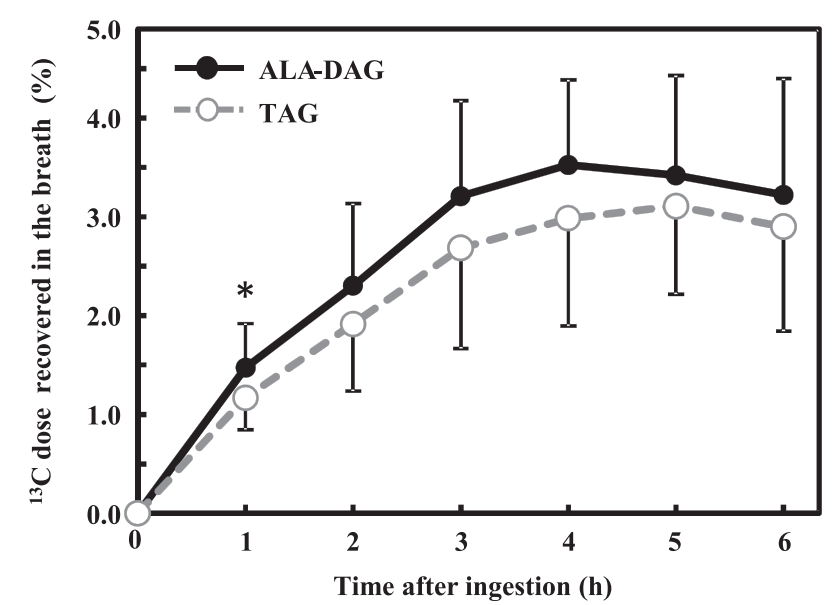

Table 2 Physical status of the subjects after the treatment with either TAG or ALA-DAG.

\begin{tabular}{lcc}
\hline \multicolumn{1}{c}{ Parameter } & TAG & ALA-DAG \\
\hline Sex $(\mathrm{M} / \mathrm{F})$ & \multicolumn{2}{c}{$11 / 5$} \\
Age (year) & \multicolumn{2}{c}{$49 \pm 9$} \\
Body weight $(\mathrm{kg})$ & $70.8 \pm 8.9$ & $70.4 \pm 8.6$ \\
Body mass index $\left(\mathrm{kg} / \mathrm{m}^{2}\right)$ & $26.0 \pm 2.0$ & $25.8 \pm 2.0$ \\
Body fat ratio $(\%)$ & $29.2 \pm 7.5$ & $28.9 \pm 7.6^{*}$ \\
Fat mass $(\mathrm{kg})$ & $20.4 \pm 4.4$ & $20.0 \pm 4.3^{*}$ \\
Fat-free mass $(\mathrm{kg})$ & $50.4 \pm 9.7$ & $50.4 \pm 9.7$ \\
Waist $(\mathrm{cm})$ & $92.4 \pm 6.2$ & $92.1 \pm 6.3$ \\
\hline Mean $\pm \mathrm{SD} ; \mathrm{n}=16 .{ }^{*}, p<0.05$ between & TAG and ALA- \\
DAG. & &
\end{tabular}

\section{DISCUSSION}

In the present study, to gain further insight into the mechanism underlying the anti-visceral obesity effect of ALA-DAG, the recovery rate of ${ }^{13} \mathrm{C}$-labelled dietary fat to ${ }^{13} \mathrm{CO}_{2}$ in the breath was assessed as an indicator of dietary fat oxidation during either ALA-DAG or TAG treatment. The ALA-DAG treatment enhanced dietary fat utilization as energy compared to the TAG treatment in humans. Taken together with our previous data showing increased postprandial total fat oxidation and energy expenditure after treatment with ALA-DAG ${ }^{11)}$, these findings suggest that ALA-DAG treatment induces up-regulation of dietary fat metabolism and contributes to prevent visceral obesity. Indeed, significantly decreased fat mass (Table 2) and significantly increased dietary fat oxidation(Fig. 1) were both observed in this present study.

Repeated consumption of ALA-DAG enhances $\beta$-oxidation in the small intestine ${ }^{12)}$ and liver ${ }^{13)}$ in rodents.

\section{B}

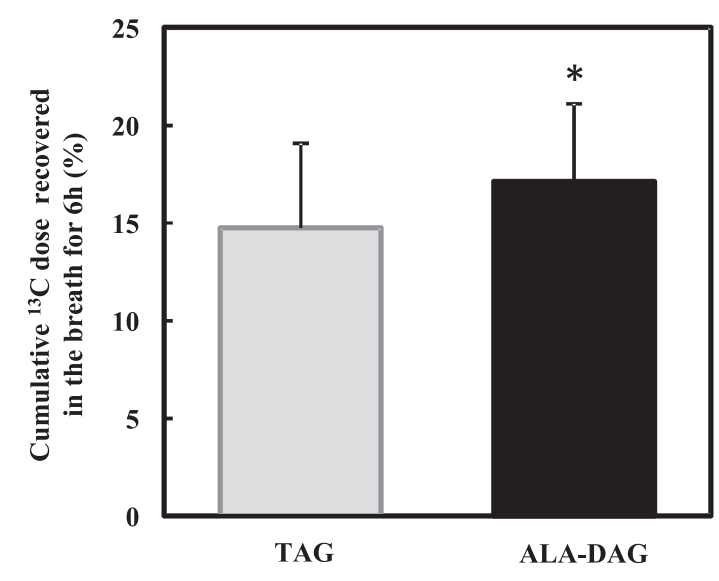

Fig. $1{ }^{13} \mathrm{C}$ recovery in the breath for up to $6 \mathrm{~h}(\mathrm{~A})$, and its cumulative value (B). Time zero corresponds to the ingestion time of the test meal containing ${ }^{13} \mathrm{C}$-triolein. Data are presented as means $\pm \mathrm{SD}$. ${ }^{*}, p<0.05$ between TAG and ALA-DAG. 
Although the precise mechanism by which ALA-DAG consumption stimulates gene expression is not clear, it may be related to the metabolic pathway of ALA-DAG. In general, the TAG structure is hydrolyzed by lipase to 2-monoacylglycerol (MAG) and fatty acids and then resynthesized into TAG in the intestinal mucosa cells. In contrast, the DAG structure is hydrolyzed to 1-(or 3) MAG and fatty acids. Because 1-MAG is not easily resynthesized to TAG in the small intestine ${ }^{19,20)}$, the 1 -MAG and free fatty acids may remain in the intestinal mucosa cells after DAG consumption $^{21)}$. Omega 3 fatty acids, such as ALA, eicosapentaenoic acid, and docosahexaenoic acid increase the gene expressions including farnesoid $\mathrm{X}$ receptor and peroxisome proliferator-activated receptor compared to other fatty acids, such as saturated and omega 6 fatty acids ${ }^{22)}$, suggesting that the free ALA derived from ALA-DAG activates fat oxidation and suppress fat synthesis, particularly in the small intestine and the liver. Thus, DAG and ALA can synergistically affect fat oxidation. Indeed, Murase et al. suggested that $\beta$-oxidation is higher in rodents fed ALA-DAG than in those fed ALA-TAG ${ }^{12)}$. Additionally, ALA-DAG can enhance fat oxidation at $25 \%$ of the dose compared to oleic and linolenic acid-riched $\mathrm{DAG}^{16)}$.

A limitation of the present study is that the ALA-DAG shortbread contained more ALA than the control TAG shortbread because we did not adjust the fatty acids component. Therefore, we cannot determine whether the DAG structure or the fatty acid composition was the major factor inducing the ALA-DAG effects. Additional studies with a similar fatty acid composition are required.

\section{CONCLUSION}

Treatment with ALA-DAG for 14-d enhanced the utilization of dietary fat as energy in healthy humans. This result indicates that ALA-DAG consumption has beneficial effects for preventing visceral obesity by upregulating dietary fat metabolism.

\section{ACKNOWLEDGMENTS}

We thank Dr. Masanobu Hibi, Mr. Takuya Wakisaka and Dr. Tohru Yamaguchi for technical and scientific advice regarding this study. We thank Dr. Haruo Nakamura (Mitsukoshi Health and Welfare Foundation) for instruction and advice regarding the study design, protocol, and discussion.

\section{CONFLICT OF INTEREST}

This study was financially supported by Kao Corpora- tion. ALA-DAG was prepared using equipment owned by Kao Corporation. Study management, sample collection, and data analysis were performed independently, and thus there are no conflicts of interest that would affect the study results.

\section{References}

1) Fujioka, S.; Matsuzawa, Y.; Tokunaga, K.; Tarui, S. Contribution of intra-abdominal fat accumulation to the impairment of glucose and lipid metabolism in human obesity. Metabolism 36, 54-59 (1987).

2) Kanai, H.; Matsuzawa, Y.; Kotani, K.; Keno, Y.; Kobatake, T.; Nagai, Y.; Fujioka, S.; Tokunaga, K.; Tarui, S. Close correlation of intra-abdominal fat accumulation to hypertension in obese women. Hypertension 16, 484-490 (1990).

3) Nakamura, T.; Tokunaga, K.; Shimomura, I.; Nishida, M.; Yoshida, S.; Kotani, K.; Islam, A. H.; Keno, Y.; Kobatake, T.; Nagai, Y.; Fujioka, S.; Tarui, S.; Matsuzawa, Y. Contribution of visceral fat accumulation to the development of coronary artery disease in non-obese men. Atherosclerosis 107, 239-246 (1994).

4) Flatt, J. P.; Ravussin, E.; Acheson, K. J.; Jéquier, E. Effects of dietary fat on postprandial substrate oxidation and on carbohydrate and fat balances. J. Clin. Invest. 76, 1019-1024 (1985).

5) Kien, C. L.; Bunn, J. Y.; Ugrasbul, F. Increasing dietary palmitic acid decreases fat oxidation and daily energy expenditure. Am. J. Clin. Nutr. 82, 320-326 (2005).

6) Dulloo, A. G.; Fathi, M.; Mensi, N.; Girardier, L. Twenty-four-hour energy expenditure and urinary catecholamines of humans consuming low-to-moderate amounts of medium-chain triglycerides: a dose-response study in a human respiratory chamber. Eur. J. Clin. Nutr. 50, 152-158(1996).

7) Kamphuis, M. M.; Mela, D. J.; Westerterp-Plantenga, M. S. Diacylglycerols affect substrate oxidation and appetite in humans. Am. J. Clin. Nutr. 77, 1133-1139 (2003).

8) Takei, A.; Katsuragi, Y.; Abe, C.; Mori, K.; Takeda, Y.; Seo, Y.; Takase, H.; Takahashi, H.; Tohata, M.; Chikama, A.; Fumoto, S.; Meguro, S.; Komine, Y.; Nagao, T.; Hase, T.; Tokimitsu, I.; Shimasaki, H.; Itakura, H. Effects of dietary alpha-linolenic acid-rich diacylglycerol on body fat in man (1): Lowering effect on body fat. $J$. Oleo Sci. 50, 735-746(2001).

9) Katsuragi, Y.; Takeda, Y.; Abe, C.; Mori, K.; Toi, T.; Takei, A.; Shimasaki, H.; Itakura, H. Effects of dietary alpha-linolenic acid rich diacylglycerol on body fat in man (2): Effects on resting metabolism and fat metabolism. J. Oleo Sci. 50, 747-752 (2001).

10) Saito, S.; Fukuhara, I.; Osaki, N.; Nakamura, H.; Kat- 
suragi, Y. Consumption of alpha-linolenic acid-enriched diacylglycerol reduces visceral fat area in overweight and obese subjects: a randomized, double-blind controlled, parallel-group designed trial. J. Oleo Sci. 65, 603-611 (2016).

11) Ando, Y.; Saito, S.; Oishi, S.; Yamanaka, N.; Hibi, M.; Osaki, N.; Katsuragi, Y. Alpha linolenic acid-enriched diacylglycerol enhances postprandial fat oxidation in healthy subjects: A randomized double-blind controlled trail. J. Oleo Sci. 65, 685-691 (2016).

12) Murase, T.; Nagasawa, A.; Suzuki, J.; Wakisaka, T.; Hase, T.; Tokimitsu, I. Dietary alpha-linolenic acid-rich diacylglycerols reduce body weight gain accompanying the stimulation of intestinal beta-oxidation and related gene expressions in C57BL/KsJ-db/db mice. $J$. Nutr. 132, 3018-3022 (2002).

13) Murase, T.; Aoki, M.; Tokimitsu, I. Supplementation with alpha-linolenic acid-rich diacylglycerol suppresses fatty liver formation accompanied by an up-regulation of beta-oxidation in Zucker fatty rats. Biochem. Biophys. Acta 1733, 224-231 (2005).

14) Watanabe, H.; Yamaguchi, T.; Onizawa, K.; Osaki, N.; Harada, U.; Matsuo, N.; Tokimitsu, I.; Shimasaki, H.; Itakura, $\mathrm{H}$. Effects of alpha-linolenic acid-rich diacylglycerol intake on the oxidation of dietary fats in rats. $J$. Oleo Sci. 50, 839-842 (2001).

15) Watanabe, T.; Shimizu, M.; Sugiura, M.; Sato, M.; Kohori, J.; Yamada, N.; Nakanishi, K. Optimization of reaction conditions for the production of DAG using immobilized 1,3-regiospecific lipase lipozyme RM IM. J. Am. Oil Chem. Soc. 80, 1201-1207 (2003).

16) Hibi, M.; Takase, H.; Yasunaga, K.; Yamaguchi, T.; Shii- ba, D.; Saito, S.; Yokoyama, R.; Kudo, N.; Katsuragi, Y.; Meguro, S.; Shimizu, A.; Tokimitsu, I. Greater fat oxidation with diacylglycerol oil consumption for 14 days compared with triacylglycerol oil consumption in overweight men and women. Int. J. Obes. (Lond.) 32, 1841-1847 (2008).

17) Beppu, F.; Asanuma, M.; Kawamura, Y.; Nagai, T.; Yoshinaga, K.; Mizobe, H.; Kojima, K.; Kasatani, S.; Nagao, K.; Kubo, A.; Kanda, J.; Gotoh, N. Trans-octadecenoic acid positional isomers have different accumulation and catabolism properties in mice. J. Oleo Sci. 64, 1159-1167(2015).

18) Beppu, F.; Konno, K.; Kawamatsu, T.; Nagai, T.; Yoshinaga, K.; Mizobe, H.; Kojima, K.; Watanabe, H.; Gotoh, $\mathrm{N}$. Comparison of catabolic rates of ${ }^{13} \mathrm{C}$-labeled palmitic acid bound to the alpha and beta positions of triacylglycerol using $\mathrm{CO}_{2}$ expired from mice. Eur. J. Lipid Sci. 117, 718-723(2015).

19) Osaki, N.; Meguro, S.; Yajima, N.; Matsuo, N.; Tokimitsu, I.; Shimasaki, H. Metabolites of dietary triacylglycerol and diacylglycerol during the digestion process in rats. Lipids 40, 281-286 (2005).

20) Kondo, H.; Hase, T.; Murase, T.; Tokimitsu, I. Digestion and assimilation features of dietary DAG in the rat small intestine. Lipids 38, 25-30 (2003).

21) Yanagita, T.; Ikeda, I.; Wang, Y. M.; Nakagiri, H. Comparison of the lymphatic transport of radiolabeled 1,3-dioleoylglycerol and trioleoylglycerol in rats. Lipids 39, 827-832 (2004).

22) Davidson, M. H. Mechanisms for the hypotriglyceridemic effect of marine omega-3 fatty acids. Am. J. Cardiol. 98, 27i-33i(2006). 